\title{
RETRACTED ARTICLE: Reviewing big data based mental health education process for promoting education system
}

\author{
Kai Wang ${ }^{1} \cdot$ Yue-e Zhou ${ }^{2} \cdot$ Jia-xin $\mathrm{Xu}^{2} \cdot$ Guang Yang $^{1}$ \\ Published online: 3 June 2020 \\ (C) Springer Science+Business Media, LLC, part of Springer Nature 2020
}

The Editor-in-Chief has retracted this Article. The article was accepted as part of a guest-edited special issue in Current Psychology. Before the special issue was finalized, the Editor-in-Chief detected problems with editorial handling and peer review and decided not to proceed with the special issue. Post publication peer review found the following issues.

- The methods section provides no clear idea of what the study aims to investigate.

- It seems the study aims to evaluate the effectiveness of public school educational programs relative to addressing student's mental health disorders but the mental health disorders are not specified.

- The methods section does not offer a clear description of the research methodology.

- There is no section clarifying the means used to analyze data.

- The methods employed are not sound.

- Basic demographic data such as a description of participants seems to be missing.

Guang Yang

yangguang1406@sina.com

Kai Wang

beihuawk@126.com

Yue-e Zhou

3263124891@qq.com

Jia-xin Xu

1025476175@qq.com

1 College of Computer Science and Technology, Beihua University, Jilin City, China

2 Software Professional Level 18 ,College of Computer Science and Technology, Beihua University, Jilin City, China
- Grounded theory and the map reduction technique are introduced but poorly described.

- The conclusions are not clearly stated.

The Editor-in-Chief therefore no longer has confidence in the reliability of the work presented.

None of the authors of this paper have responded to any correspondence from the Editor about this retraction.

The online version of this article contains the full text of the retracted article as Supplementary Information.

Supplementary Information The online version of this article (https://doi. org/10.1007/s12144-020-00800-6) contains supplementary material, which is available to authorized users. 